\title{
Olfactory Bulb Gamma Oscillations Are Enhanced with Task Demands
}

\author{
Jennifer Beshel, ${ }^{1}$ Nancy Kopell, ${ }^{2}$ and Leslie M. Kay ${ }^{1}$ \\ ${ }^{1}$ Department of Psychology and Institute for Mind and Biology, The University of Chicago, Chicago, Illinois 60637, and ${ }^{2}$ Department of Mathematics and \\ Statistics and Center for BioDynamics, Boston University, Boston, Massachusetts 02215
}

Fast oscillations in neural assemblies have been proposed as a mechanism to facilitate stimulus representation in a variety of sensory systems across animal species. In the olfactory system, intervention studies suggest that oscillations in the gamma frequency range play a role in fine odor discrimination. However, there is still no direct evidence that such oscillations are intrinsically altered in intact systems to aid in stimulus disambiguation. Here we show that gamma oscillatory power in the rat olfactory bulb during a two-alternative choice task is modulated in the intact system according to task demands with dramatic increases in gamma power during discrimination of molecularly similar odorants in contrast to dissimilar odorants. This elevation in power evolves over the course of criterion performance, is specific to the gamma frequency band $(65-85 \mathrm{~Hz})$, and is independent of changes in the theta or beta frequency band range. Furthermore, these high amplitude gamma oscillations are restricted to the olfactory bulb, such that concurrent piriform cortex recordings show no evidence of enhanced gamma power during these high-amplitude events. Our results display no modulation in the power of beta oscillations (15-28 Hz) shown previously to increase with odor learning in a Go/No-go task, and we suggest that the oscillatory profile of the olfactory system may be influenced by both odor discrimination demands and task type. The results reported here indicate that enhancement of local gamma power may reflect a switch in the dynamics of the system to a strategy that optimizes stimulus resolution when input signals are ambiguous.

Key words: oscillations; gamma oscillations; perceptual learning; sensory acuity; beta oscillations; olfactory bulb; piriform (pyriform)

\section{Introduction}

The chemotopic organization of vertebrate and insect olfactory systems (Mori et al., 1992; Johnson et al., 2004) suggests that disambiguating odors occupying overlapping glomerular regions, or molecularly similar odors, offers a different perceptual challenge than odors represented by unique cell populations, or molecularly dissimilar odors (Linster et al., 2001; Cleland and Linster, 2002). The coordinated activity of cell populations, reflected by regular oscillations of local field potentials (LFPs), has been proposed as a mechanism to optimize stimulus representation, particularly in the case of noisy input signals (Konig et al., 1995; Stopfer et al., 1997; Friedrich and Laurent, 2001; Friedrich et al., 2004). Power and periodicity of olfactory bulb (OB) LFP oscillations varies with the degree that mitral cells couple to the population rhythm (Gray and Skinner, 1988; Eeckman and Freeman, 1990). These precise firing patterns may aid stimulus resolution by increasing the probability of coincident firing to downstream targets, which are sensitive to synchronous or closely spaced inputs (Bower and Haberly, 1986; Perez-Orive et al., 2002, 2004).

Received March 16, 2007; revised June 23, 2007; accepted June 23, 2007.

This work was supported by a Collaborative Research in Computational Neuroscience grant [National Institute on Deafness and Other Communication Disorders (NIDCD) Grant R01DC007995 (L.M.K. and N.K.)] and NIDCD Predoctoral Fellowship F31DC008467 (J.B.). We thank Claire Martin for numerous helpful discussions and comments on this manuscript.

Correspondence should be addressed to Leslie M. Kay, Institute for Mind and Biology, 940 East 57th Street, Chicago, IL 60637. E-mail: Ikay@uchicago.edu.

DOI:10.1523/JNEUROSCI.1199-07.2007

Copyright $\odot 2007$ Society for Neuroscience $\quad$ 0270-6474/07/278358-08\$15.00/0
High-frequency oscillations have been correlated previously with odor discrimination demand in two phylogenetically different olfactory systems: the antennal lobe of pharmacologically manipulated honeybees and the OB of mutant mice. Stopfer et al. (1997) showed that the same manipulation that disrupts oscillations $(\sim 20 \mathrm{~Hz})$ in the honeybee olfactory system results in abnormally high behavioral generalization to a single odorant molecularly similar to a single reinforced odorant. This is indirect support for the role of oscillations in fine discrimination, because concurrent behavioral and electrophysiological recordings were not done; it is not known whether intact honeybees produce these oscillations in a behavioral context. Nusser et al. (2001) showed that $\beta 3$ knock-out mice have enhanced gamma oscillations (65$115 \mathrm{~Hz}$ ) and generalize less to odorants closely related to a single reinforced odorant than their wild-type littermates. However, this deletion of a class of $\mathrm{GABA}_{\mathrm{A}}$ receptors was not isolated to the $\mathrm{OB}$, and the mice displayed gross phenotypic abnormalities (Homanics et al., 1997). It is thus unclear whether discrimination enhancement resulted from nonspecific behavioral differences between knock-out and wild-type mice or increases in gamma power. Again, concurrent neural and behavioral recordings were not performed. Because evidence of a functional role for these oscillations in odor discrimination comes from severely disrupted systems, the question of whether they are relevant to resolving odors during normal functioning remains unanswered. Additionally, because previous support is indirect, little is known about how these oscillations might develop during the process of odor discrimination learning or performance. 
To address this, we coupled operant behavior with neural recording in the olfactory system of unmanipulated rats to determine whether they dynamically change the level of population synchrony to adjust to task demands. Because changes in these oscillations selectively affect discrimination of molecularly similar odor pairs, we hypothesized that gamma power would be upregulated selectively for fine discrimination compared with coarse discrimination. To test this, we used a two-alternative choice odor discrimination paradigm and manipulated task demand with multiple "coarse" and "fine" odor pairings.

\section{Materials and Methods}

Four adult male Sprague Dawley rats were implanted with electrodes in the olfactory bulb and piriform cortex using stereotaxic coordinates as a guide and stimulation of the lateral olfactory tract for precise positioning, as reported previously (Kay and Freeman, 1998; Kay, 2005). After recovery, rats were maintained at $85 \%$ of their ad libitum weight with restricted food intake and unlimited water. All surgical and behavioral procedures were done with approval and oversight by the University of Chicago Institutional Animal Care and Use Committee, according to Association for Assessment and Accreditation of Laboratory Animal Care guidelines.

Behavior. The rats were trained to initiate each trial by pressing a lever located at the rear of the chamber, which illuminated the chamber house light. Rats then poked their noses into the odor port located at the front of the chamber. Triggered by the infrared beam in the nose-poke operandum, either odor was pseudorandomly delivered for $1.5 \mathrm{~s}$. Odors were generated in removable glass test tubes by bubbling air $(100 \mathrm{ml} / \mathrm{min})$ through a column of pure liquid odorant and injecting the odorized air into a carrier air stream $(400 \mathrm{ml} / \mathrm{min})$ via a computer-controlled olfactometer achieving $\sim 20 \%$ saturated vapor. After odor delivery, levers on either side of the odor port extended. A lever press to the correct side delivered a single sucrose pellet reward ( $85 \%$ reinforcement schedule; 40 mg pellet; BioServe, Frenchtown, NJ). Incorrect responses (lever press to the wrong side) resulted in immediate extinguishing of the house light and an extra $4 \mathrm{~s}$ delay until the next trial could be initiated. Each session was terminated after 200 trials. Because there was trial-to-trial timing variability in when the rat chose to initiate a new trial by pressing the rear lever, session durations ranged in length from 1.5 to $2.5 \mathrm{~h}$. Task events were controlled by subroutines within Graphic State 4.0 (Coulbourn Instruments, Allentown, PA). Rats learned the task to a high degree of reliability (90-95\%) before electrode implantation. After implantation, recordings were obtained using the training odor set (amyl acetate and ethyl 2-methylbutyrate) until performance reached presurgery levels. Experimental odor pairs were then introduced, and criterion performance for each odor set was set at 2 consecutive days of $\geq 70 \%$ correct choice. For purposes of comparison across and within odorant functional groups, each rat was presented with four different odor pairs in balanced order across subjects. The functional groups included were as follows: ketones [coarse pair, butanone/nonanone (Aldrich, Milwaukee, WI); fine pair, heptanone/octanone (Aldrich)] and alcohols [coarse pair, propanol/octanol (Fisher Scientific, Fair Lawn, NJ); fine pair, hexanol/heptanol (ICN Biomedicals, Aurora, OH)].

Electrophysiology. Local field potentials in the olfactory bulb and piriform cortex were recorded differentially using stainless steel Formvarcoated electrodes $(100 \mu \mathrm{m})$ with reference to a skull screw. Neural data along with behavioral event markers were recorded with a Neuralynx (Tucson, AZ) Cheetah system. Signals were sampled at $2016 \mathrm{~Hz}$ and amplified $(2000 \times)$, and analog filters were set at $1-475 \mathrm{~Hz}$. A unity gain preamplifier head stage (NB Labs, Denison, TX) was used for signal conditioning. Signals were digitally filtered and analyzed off-line with IGOR Filter Design Laboratory 4.01 and IGOR Pro 5.0 (WaveMetrics, Lake Oswego, OR). Statistics were performed using SPSS 10.0 (SPSS, Chicago, IL).

Data analysis. The odor delivery period was extracted from filtered data (gamma, 35-115 Hz; beta, $15-28 \mathrm{~Hz}$; theta, $4-10 \mathrm{~Hz}$ ) and aligned by the peak of the sensory evoked potential (EP) in the raw data by using synchronized behavioral markers (nose poke) coupled with an iterative process from the session averaged EP to identify the EP in individual trials. Extracted data were normalized by the SD of the presession LFP signal ( $\sim 10 \mathrm{~s}$, filtered $10-150 \mathrm{~Hz}$ to limit signal variance attributable to movement artifact) before estimating the spectra to allow comparisons across sessions and rats. Autospectra were estimated by first applying a 512 point Hamming taper to each data window and then taking the fast Fourier transform and computing the spectrum. Seven half-overlapping 512 point windows per $1100 \mathrm{~ms}$ time segment were used to obtain the averaged power spectrum for the odor delivery period. Averaged gamma power for odor responses (see Fig. $4 A, B$ ) was obtained by integrating the band between 65 and $85 \mathrm{~Hz}$. We used this band to avoid spurious increases attributable to line noise in some sessions. Averaged beta and theta power for odor responses was obtained by integrating the band between 15-28 and 4-10 Hz, respectively. Averaged number of sniffs per odor delivery period was obtained by counting automatically detected theta peaks with amplitude greater than the SD of the presession LFP filtered between 4 and $10 \mathrm{~Hz}$. For the pre-odor period, estimates were obtained as above using the $1100 \mathrm{~ms}$ time segment before the sensory EP. Dynamic power spectra centered on odor delivery (see Fig. $3 A-D$ ) were estimated for each 1024 point window stepped by $250 \mathrm{~ms}$. For blockwise analysis (see Figs. 5, 6), the spectra were collected for the odor delivery period across 20 consecutive trials, integrated $(65-85 \mathrm{~Hz})$, and averaged. Statistical comparisons were made by two- and three-factor withinsubjects repeated-measures ANOVAs for session data. Factors included the following: demand (coarse, fine); functional group (ketone, alcohol); performance (naive, criterion); and chain length (four levels corresponding to each odorant carbon chain length). One-way ANOVAs were used to assess blockwise differences between the pre-odor and odor period for each animal. The significance level was set at $p<0.05$.

\section{Results}

All rats learned to discriminate the odor pairs and reached criterion performance in one to six sessions (one session per day). Discrimination of molecularly dissimilar odor pairs was accomplished relatively quickly (Fig. 1C) (sessions to criterion \pm SEM; ketones, $1 \pm 0.41$; alcohols, $1.5 \pm 0.65)$. In contrast, fine discrimination took somewhat longer to achieve (Fig. 1C) [ketones, $3.75 \pm$ 1.38 sessions; alcohols, $1.75 \pm 0.85$ sessions; $f_{\text {demand }}(1,3)=27.0, p=$ $0.014 ; f_{\text {demand }} \times$ functional group $\left.(1,3)=1.271, \mathrm{NS}\right]$. Once animals reached criterion, there was no difference in performance between demand conditions over the course of a session $\left[f_{\text {demand }}(1,3)=0.58\right.$, NS; coarse, $83 \%$; fine, $82 \%$ ]. This was true of both functional groups $\left[f_{\text {demand }} \times\right.$ functional group $(1,3)=0.528$, NS; ketones: coarse, $82 \%$; fine, $78 \%$; alcohols: coarse, $83 \%$; fine, $85 \%$ ].

\section{Gamma power increases during fine odor discrimination}

To test the hypothesis that fine odor discrimination is accompanied by higher population synchrony in the gamma frequency band, we first test the power of the olfactory bulb gamma band during fine versus coarse odor discrimination. We find that gamma power increases with task demands and is readily observable in the raw olfactory bulb LFP signal during the odor delivery period (Fig. $2 A, B$ ). These bursts of oscillations last between 0.5 and $1 \mathrm{~s}$ and directly follow a sensory evoked potential. Spectral analysis reveals that the dramatic increase occupies a narrow band of frequencies $(60-85 \mathrm{~Hz})$ within the reported range for classical odor-associated gamma oscillations in the rat (Bressler and Freeman, 1980; Kay, 2003) (Fig. 2C). To be conservative, we limited our analysis to frequencies between 65 and $85 \mathrm{~Hz}$ to prevent the possible contribution of $60 \mathrm{~Hz}$ line noise to the observed differences. Comparisons between criterion sessions ( $>70 \%$ correct) of either fine or coarse odor discrimination show that gamma power is significantly greater during discrimination of molecularly similar than molecularly dissimilar odorants [fine $>$ coarse, $\left.f_{\text {demand }}(1,3)=11.621 ; p=0.042\right]$. The magnitude of this difference in power varies as a function of time and is discussed in greater depth below. 
A

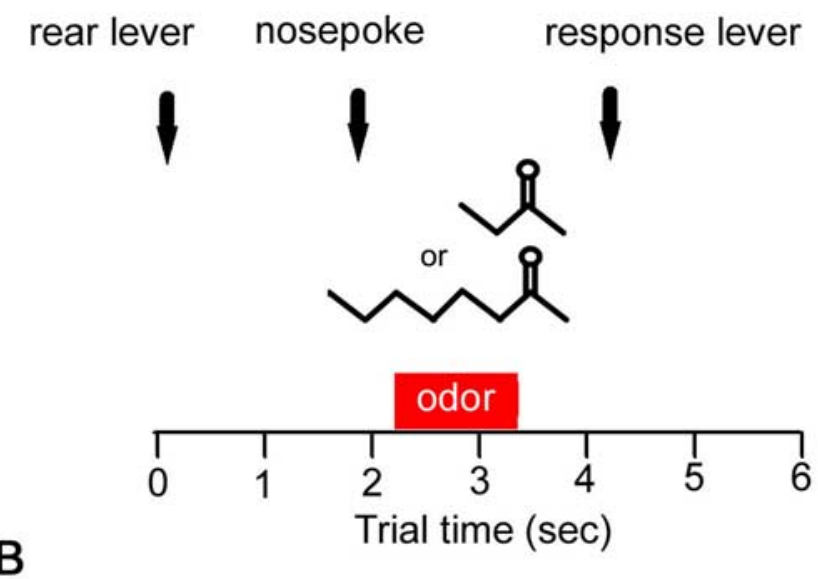

rear lever nosepoke response lever

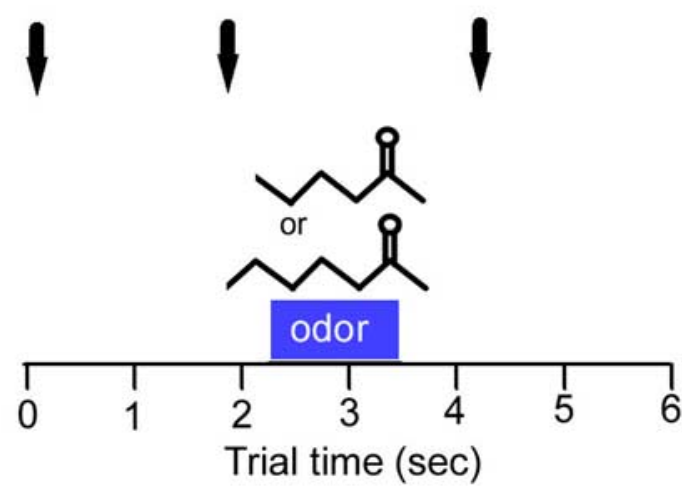

C

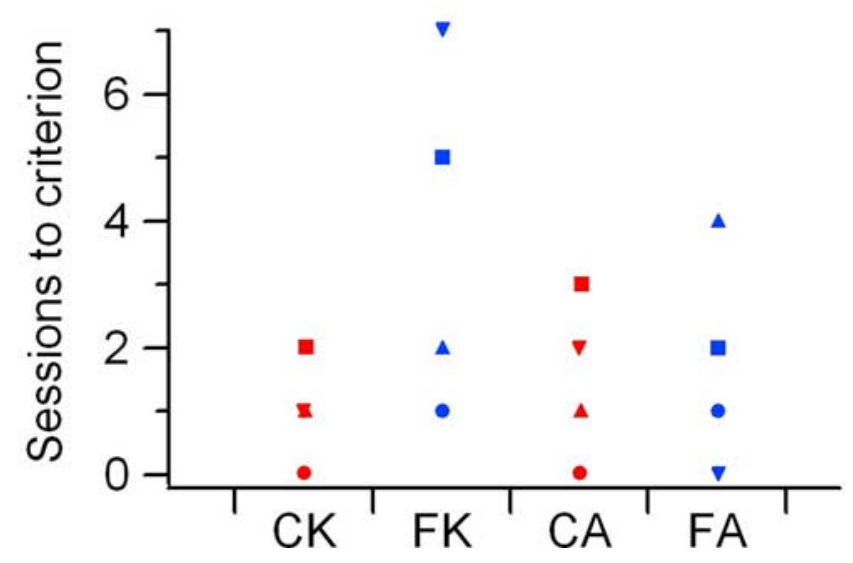

Figure 1. Rats were trained on a two-alternative choice odor discrimination task in which a correct response to either odor was rewarded (see Materials and Methods). $\boldsymbol{A}, \boldsymbol{B}$, For coarse discrimination, the odors differed by at least four carbons in chain length $(A)$, and, for fine discrimination, the odors differed by only one carbon (B). $\boldsymbol{C}$, Criterion performance (2 consecutive days of $\geq 70 \%$ correct choice) generally took longer to attain for fine discrimination (blue symbols) compared with coarse discrimination (red symbols) with the ketone odor pairs. Sessions to criterion for fine and coarse alcohol pairs were commensurate. Each symbol denotes one rat. CK, Coarse ketones; FK, fine ketones; $C A$, coarse alcohols; FA, fine alcohols.

These oscillations were present regardless of the functional groups of the odorants used in the pairings as evidenced by the dynamic power spectra (Fig. 3). Both ketone and alcohol functional groups showed an increase in gamma power in fine relative

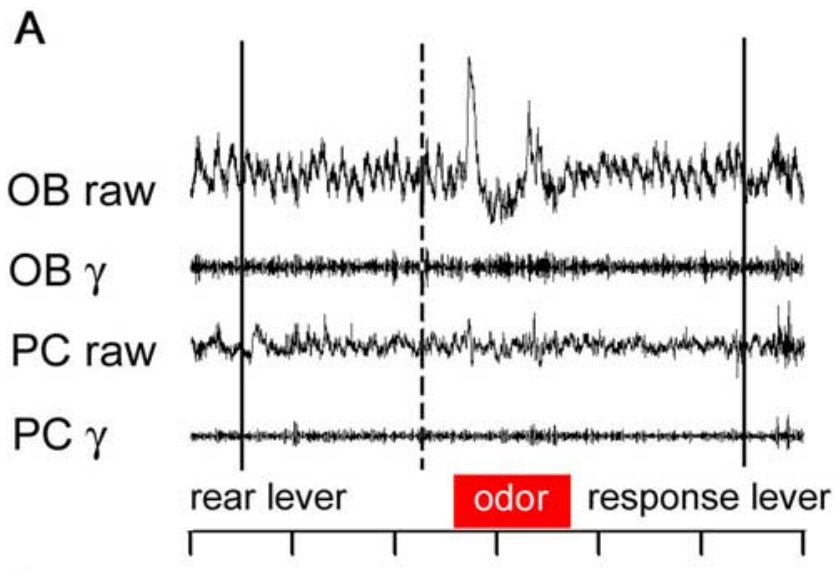

B
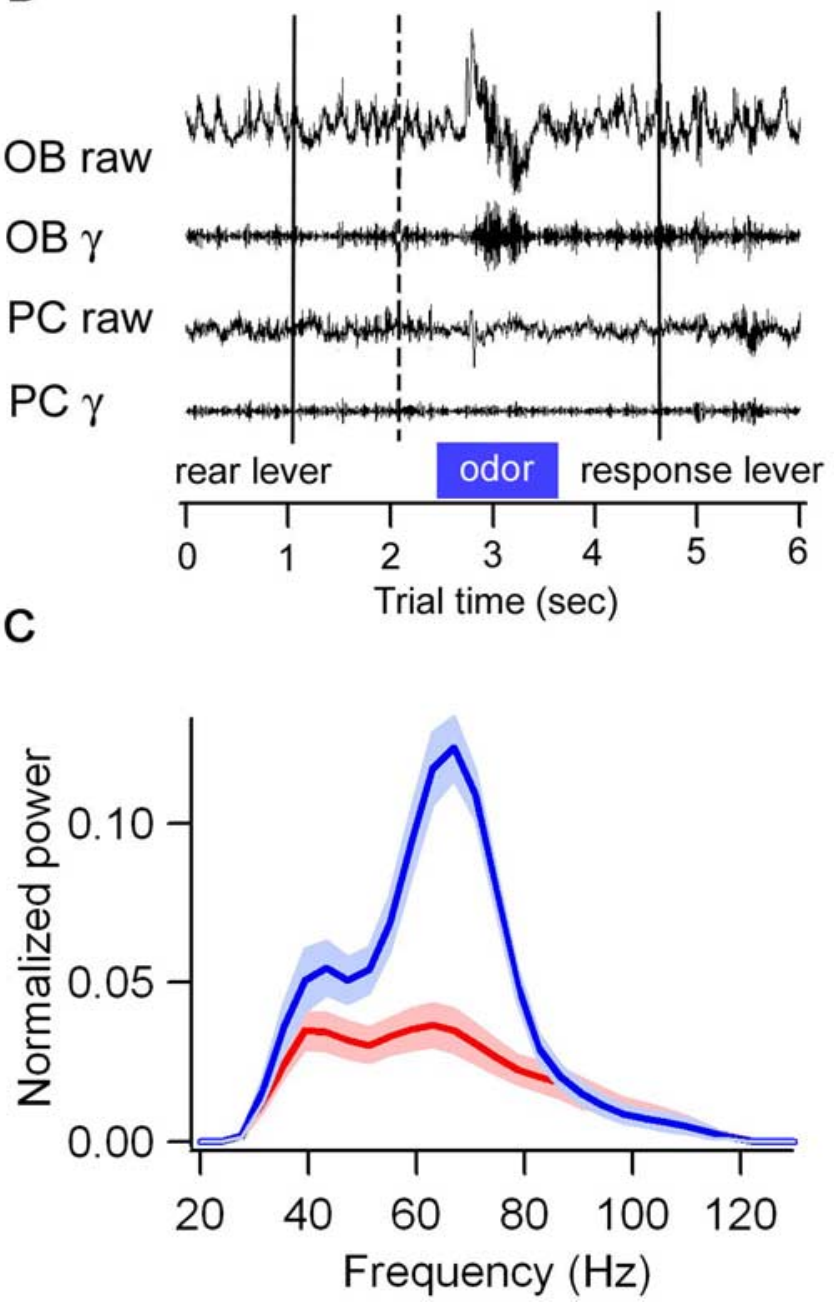

Figure 2. Fine odor discrimination elicits higher power gamma oscillatory activity in the LFP of the rat $O B$ relative to gamma power during coarse odor discrimination. $A, B$, Example of raw and filtered $(\gamma, 35-115 \mathrm{~Hz})$ LFP traces from the rat $0 B$ and piriform cortex $(\mathrm{PC})$ during one trial from a criterion session involving coarse $(\boldsymbol{A})$ and fine $(\boldsymbol{B})$ discrimination of ketones. $\boldsymbol{C}$, Power spectra (average of 20 trial block at $85 \%$ correct choice; shaded regions indicate \pm 1 SEM) for the odor delivery period during coarse (red) and fine (blue) odor discrimination (example from one rat, rf56) reveal a dramatic elevation of gamma power in the $O B$ during fine odor discrimination with reliable increases between 65 and $85 \mathrm{~Hz}$. Gamma power is given in mean \pm 1 SEM in square millivolts.

to coarse discrimination (Fig. $3 B, D$ ) [fine $>$ coarse, $f_{\text {demen }}(1,3)=$ $11.621, p=0.042 ; f_{\text {demand }} \times$ functional group $\left.(1,3)=5.834, \mathrm{NS}\right]$. The increase in gamma power was restricted to the odor sampling window, with the prestimulus period showing no difference in 


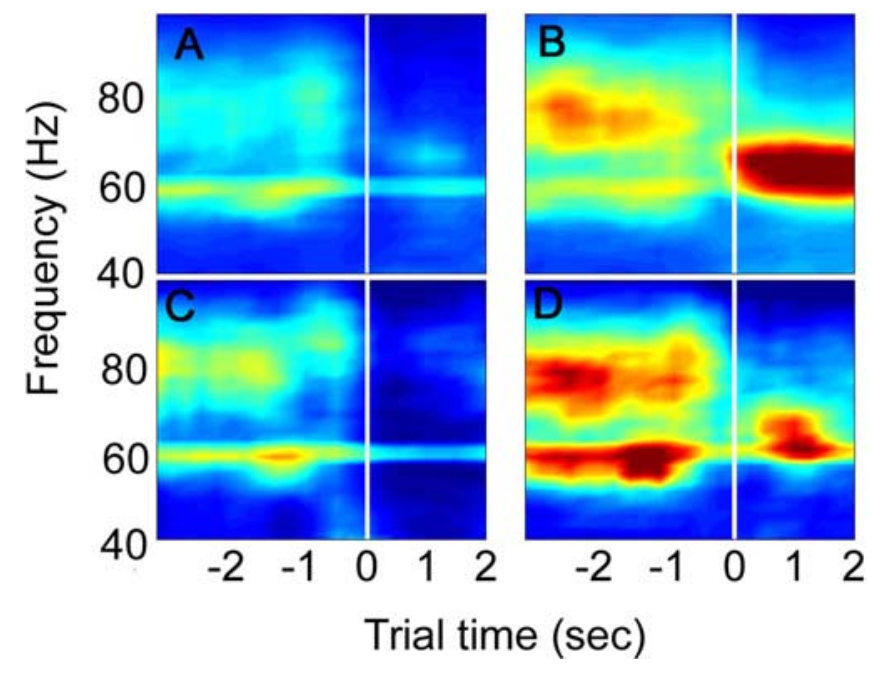

Figure 3. Dynamic power spectra show that elevation of gamma power during odor sampling is unique to fine odor discrimination for both odorant functional groups ( 0 time is the peak of the sensory evoked potential at the onset of odor sampling). A-D, Averages across 200 trials for four separate sessions from the same rat (rf73) at criterion performance for discrimination of ketones paired for coarse $(\boldsymbol{A})$ and fine $(\boldsymbol{B})$ discrimination and alcohols paired for coarse $(\boldsymbol{C})$ and fine $(\boldsymbol{D})$. Odor onset is indicated by the vertical white line at $0 \mathrm{~s}$. Color scale indicates dimensionless power and is consistent for the four plots. Increases in gamma power are restricted to the odor sampling window (large warm coloration after the vertical white line for fine odor discrimination). Although the example is suggestive, group statistics show no elevation in gamma power during the prestimulus period as a function of task demands.

power between task demand conditions across animals $\left[f_{\text {demand }}(1,3)=0.425, \mathrm{NS}\right]$. However, during odor sampling, gamma power for ketones, in circumstances of both fine and coarse discrimination, was greater than for alcohols at levels approaching significance $\left[f_{\text {functional group }}(1,3)=9.55, \mathrm{NS}, p=0.054\right.$; ketones, $0.23 \pm 0.026$; alcohols, $0.141 \pm 0.014$; mean $\pm \mathrm{SEM}]$. Such a difference may be attributable to a meaningful variation in the degree of gamma band oscillatory activity within coordinated assemblies necessary for ketone resolution, perhaps as a result of greater input overlap within ketones. Alternatively, electrodes may have been situated preferentially to detect changes in oscillations related to ketones, or the higher partial pressure of ketones in vapor form may simply produce a more intense stimulus and result in stronger oscillations. Because the level of gamma power during coarse discrimination was also lower for alcohols, this suggests that the observed baseline gamma-band difference between functional groups may not be related to their discriminability in this task.

We also considered the possibility that the increase in gamma power observed during criterion performance of fine discrimination was attributable to some inherent property of one or both of the odors used in the fine pairing or sensitization after repeated samplings of the same odors and not necessarily the context imposed by task demands. If the observed power were directly related to the odorant or a process of sensitization, we would expect differences in gamma power to be simply a function of odor sampling, independent of performance. However, in naive (first) sessions in which the animals had no previous experience with an odor set, gamma power remained low across all odor pairs; only with criterion performance did an increase in gamma power related to task demands emerge $\left[f_{\text {demand }}(1,3)=2.628, \mathrm{NS}\right.$; criterion $>$ naive, $f_{\text {demand }} \times$ performance $(1,3)=$ $46.969, p=0.006 ; f_{\text {demand }} \times$ functional group $\times$ performance $\left.(1,3)=0.807, \mathrm{NS}\right]$. To determine whether the effects observed were driven by the individual odors (e.g., butanone or nonanone) that comprised each set (e.g., coarse ketones), we compared the level of gamma power for each odor during naive and criterion performance. Like the odor set data, an interaction between the carbon chain length of individual odors and the discrimination demand imposed by their coarse or fine pairing is present only when performance is considered $\left[f_{\text {chain length }}(3,9)=1.799, \mathrm{NS} ; f_{\text {performance }} \times\right.$ chain length $(3,9)=19.073, p=0.0001]$. Pairwise comparisons reveal that gamma power did vary with chain length during naive sessions but with no consistent pattern (Fig. $4 A, B$, open circles). In the naive ketone sessions (Fig. $4 A$, open circles), butanone showed slightly higher power than nonanone, and the two odors used for fine discrimination (heptanone and octanone) did not show elevated gamma power. For alcohols, there was also a significant variation with carbon chain length, but no one odor drove the effect (Fig. $4 B$, open circles). The patterns are much more dramatic and systematic in criterion sessions (Fig. $4 A, B$, filled squares). Both odors paired for fine discrimination (heptanone and octanone in the ketone group and hexanol and heptanol in the alcohol group) had significantly higher gamma power than the odors within the same group paired for coarse discrimination [butanone or nonanone and propanol or octanol, respectively; ketones, $f_{\text {chain length }}(3,9)=1.408$, NS; $f_{\text {performance }} \times$ chain length $(3,9)=7.855, p=0.007$; alcohols, $f_{\text {chain length }}(3,9)=1.757$, NS; $f_{\text {performance }} \times$ chain length $(3,9)=4.687$, $p=0.031]$. Highly regular gamma bursts were clearly visible in the LFPs for both odors in fine odor discrimination criterion sessions and were absent in coarse odor discrimination sessions (Fig. 4C,D).

\section{Gamma power increase is unrelated to the olfactory bulb theta oscillation}

The theta frequency oscillation in the olfactory bulb LFP (4-10 $\mathrm{Hz}$ during odor sniffing) is closely related to the afferent drive from inhalation (Klingberg and Pickenhain, 1965; Macrides and Chorover, 1972; Kay, 2005). We considered whether the increase in gamma power in criterion sessions was related to differences in apparent sniffing frequency or strength of the theta oscillation. We examined theta frequency activity in the olfactory bulb during criterion performance with several measures. Because the theta band signal represents respiratory drive, the signal can be relatively asymmetric, with longer breaks $(\sim 200 \mathrm{~ms})$ between relatively brief inhalation cycles $(\sim 120 \mathrm{~ms})$. We therefore estimated the number of sniffs by counting theta oscillation peaks within the same time window used for the gamma power $(1.1 \mathrm{~s})$. The number of theta peaks for each discrimination condition was very similar [fine, 5.68 peaks; coarse, 5.40 peaks; $f_{\text {demand }}(1,3)=$ $8.136, \mathrm{NS}]$. The overall power in the theta band also did not differ between the two conditions $\left[f_{\text {demand }}(1,3)=0.316\right.$, NS $]$. Because an increase in theta power can represent either an increase in the number of peaks (smaller intersniff intervals) or in the amplitude of individual oscillations, we normalized the theta power by the number of peaks within the $1.1 \mathrm{~s}$. period, and again there was no difference between fine and coarse discrimination $\left[f_{\text {demand }}(1,3)=4.353, \mathrm{NS}\right]$.

\section{Gamma power increase is restricted to the olfactory bulb}

Outside of fast sniffing periods, large-amplitude gamma oscillations in the olfactory bulb are normally accompanied by loweramplitude gamma oscillations in the piriform cortex, apparently driven by olfactory bulb input (Kay, 2003; Martin et al., 2006). We therefore tested whether the large gamma oscillations seen in fine odor discrimination are restricted to the olfactory bulb or are also seen in the piriform cortex. During fine odor discrimination, when olfactory bulb gamma power is significantly elevated, there 

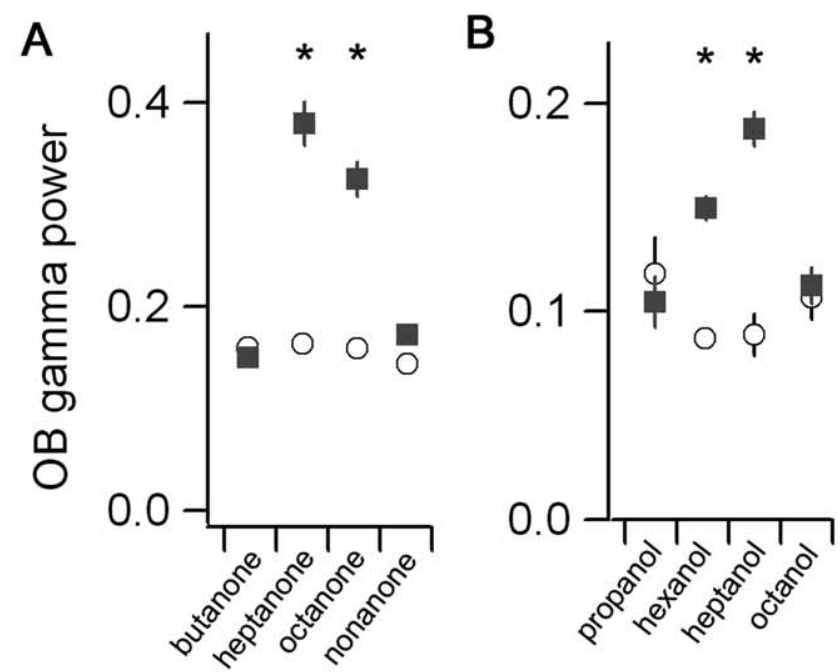

C

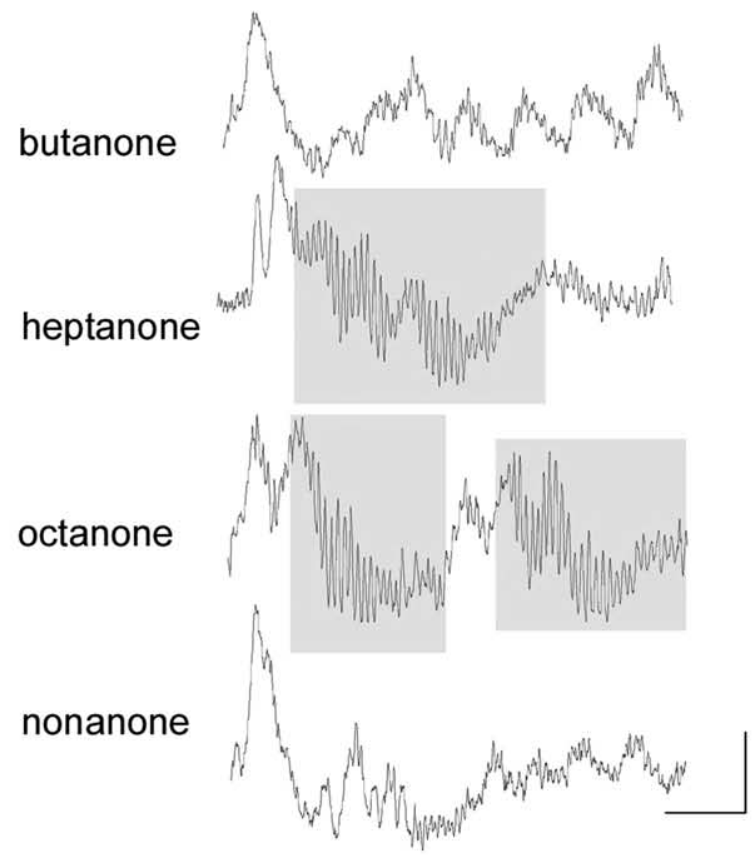

D

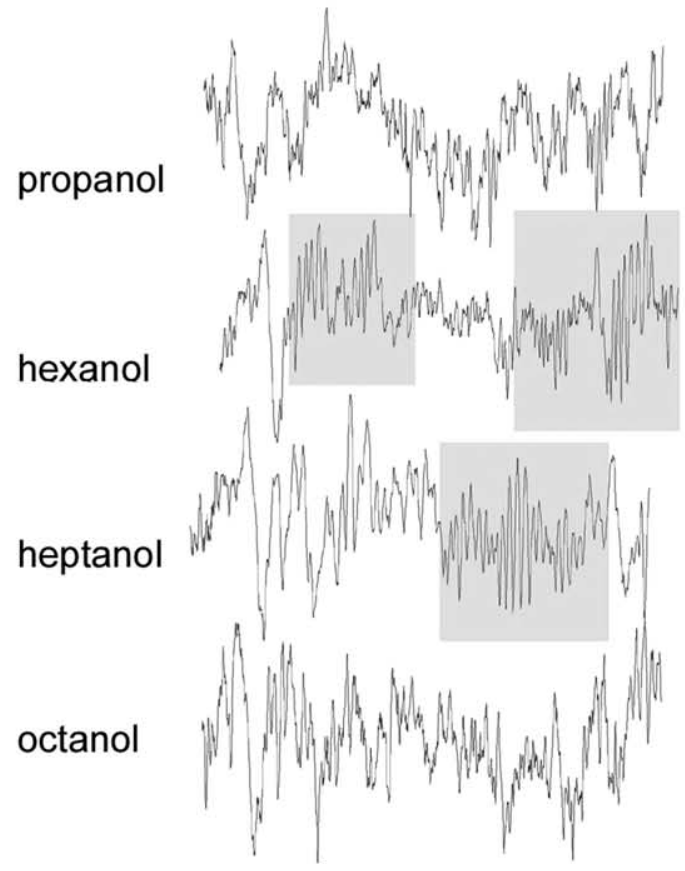

is no increase in piriform cortex gamma power compared with coarse discrimination (Fig. $2 B$ ) $\left[f_{\text {demand }}(1,3)=0.016, N S\right]$.

\section{Gamma power evolves with accurate performance}

Experience-related changes in the dynamics of olfactory system LFPs have been reported for associative and nonassociative learning in vertebrates and invertebrates, respectively (Stopfer and Laurent, 1999; Martin et al., 2004). We thus characterized changes in the odor-evoked gamma oscillations related to both performance and task demand at a finer temporal scale than the session-wise data. In particular, we examined gamma-band power in prestimulus and odor sampling periods across nonoverlapping 20-trial blocks from the naive state through two criterion performance sessions (Fig. 5). Discrimination of molecularly dissimilar odor pairs was maintained with little difference in gamma power between prestimulus and odor sampling periods across the sessions (Fig. 5A). In contrast, in fine discrimination, the first sessions showed a gamma profile similar to that seen during coarse discrimination, and performance remained poor (Fig. 5B, session 1). In criterion sessions (Fig. $5 B$, sessions 2 and 3 ), there was an initial, short-lived period of low-level gamma activity (Fig. $5 B$, downward arrow) (20-40 trials; $\sim 10-20 \mathrm{~min})$, followed by a continuous increase in power while performance remained constant and accurate. Within these first trial blocks, examination of individual trials shows that there was little elevation of gamma power relative to the pre-odor period, but there is no suppression of gamma as was often evident in the coarse discrimination case. There are also blocks in which gamma power is high but performance is below criterion. This was most common during sessions in which a rat took many more sessions to consistently reach criterion levels but also occurred intermittently during criterion sessions (Fig. 5B, upward arrow) (65\% correct). Gamma power during the prestimulus period shows no observable modulation as a function of trial block and no difference between task demand conditions. In the piriform cortex, there was no associated evolution of gamma power during odor sampling, and, in many cases, there was a decrease in gamma power during odor sniffing relative to the pre-odor period (Fig. 5).

\section{Power increase during criterion performance is restricted to} the gamma frequency band

Recent studies have shown a selective increase in beta oscillations $(15-28 \mathrm{~Hz})$ and a decrease in gamma oscillations, accompanying odor learning in a Go/No-Go odor discrimination task (Martin et al., 2004). We therefore also examined the power in the beta frequency band in both the olfactory bulb and piriform cortex during the course of learning, and we found that beta oscillations were not enhanced in fine versus coarse odor discrimination associated with reaching criterion performance (Fig. 6). In the ol-

$\leftarrow$

Figure 4. In naive sessions, the magnitude of gamma oscillatory power does not differ with respect to chemical structure. Increases in gamma power are only observable in criterion sessions and thus depend on accurate performance. Only odors paired for fine discrimination display increases in gamma power from naive (open circles) to criterion (filled squares) sessions. $\boldsymbol{A}$, $\boldsymbol{B}$, This is true of both ketones $(\boldsymbol{A})$ and alcohols $(\boldsymbol{B})$. Although power differs among individual odors in naive sessions, pairwise comparisons show that power does not vary systematically. In criterion sessions, individual odors differ in power in a manner consistent with task demands. Error bars denote \pm 1 SEM. ${ }^{*} p<0.0001$, significant increase from naive to criterion session. $C$, $D$, Example raw LFP traces from the odor sampling period from criterion sessions for ketones ( $C$ ) and alcohols $(\boldsymbol{D})$. Traces are arrayed in chain length order for each set, with the top and bottom traces forming the coarse odor pair and the two middle traces forming the fine odor pair. High-amplitude, regular gamma bursts are shaded. Calibration: $100 \mathrm{~ms}, 0.5 \mathrm{mV}$. 
A

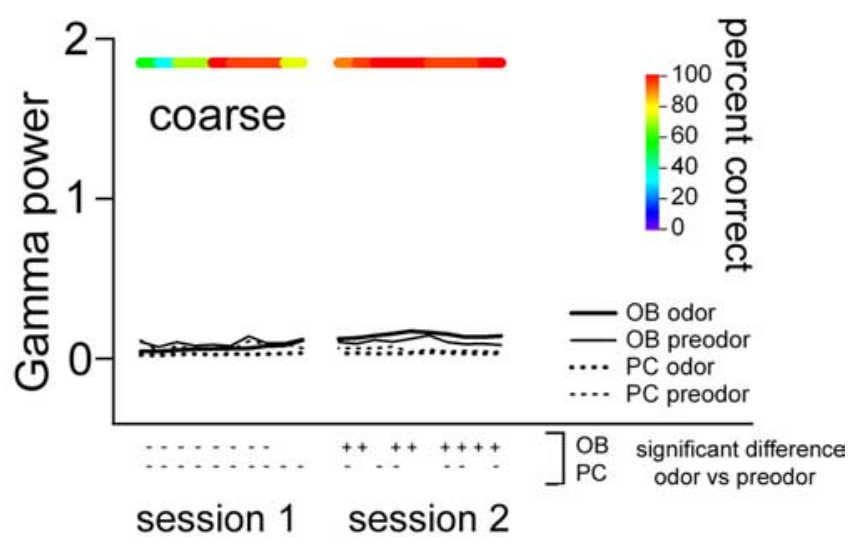

B

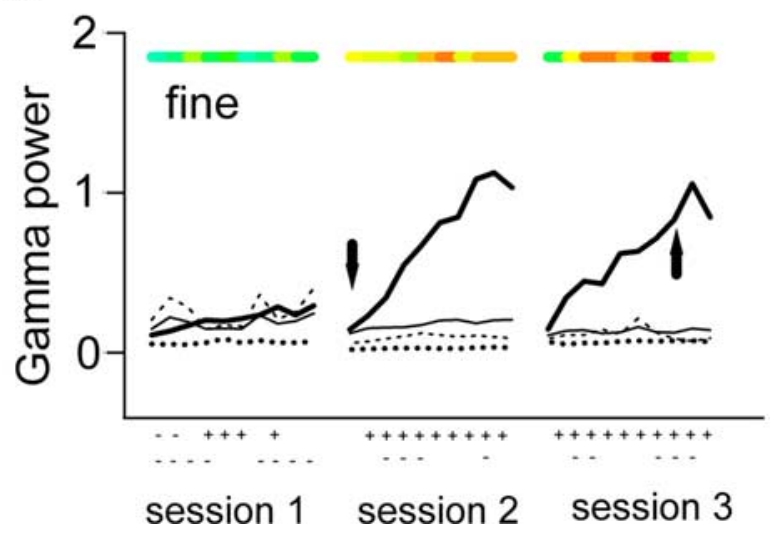

Figure 5. Gamma power during odor sampling evolves dramatically during fine discrimination criterion performance and resets at the beginning of each session. Percentage of correct trials (color bars at top) and averaged gamma band power (vertical axis) were collected across 20 trial blocks for the odor sampling period (thick solid and dashed lines) and prestimulus period (thin solid and dashed lines) for ketone discrimination acquisition (rat rf16 shown). Criterion performance was set at $70 \%$ correct. $A$, For coarse discrimination (butanone/nonanone), criterion performance was attained within the first session and maintained through the second session with little increase in gamma power during odor sampling relative to the prestimulus period. $\boldsymbol{B}$, For fine discrimination (heptanone/octanone), performance was poor for the first session with levels of gamma power similar to those observed during coarse discrimination. In the subsequent two sessions, the rat performed well and gamma power evolved from relatively low power to dramatically high power across trial blocks. The two lines located underneath each graph indicate significant differences between odor and pre-odor periods for the olfactory bulb (top) and piriform cortex (PC) (bottom); +, odor $>$ pre-odor; - , odor $<$ pre-odor; $p<0.05$; blank spaces are NS. Downward arrow signifies first trial block in which performance is high but gamma power is not elevated over pre-odor levels; upward arrow indicates a trial block with elevated gamma power but performance below criterion.

factory bulb, there was no difference in beta power between fine and coarse discrimination when data from criterion sessions were analyzed $\left[f_{\text {demand }}(1,3)=0.051, \mathrm{NS}\right]$. However, there was marginally enhanced, although not significant, beta power in the first session for fine odor discrimination sets relative to beta power during criterion performance, regardless of how many sessions were required to reach criterion performance [naive $>$ criterion, $f_{\text {demand }} \times$ performance $(1,3)=9.212$, NS, $p=$ $0.056 ; f_{\text {demand }} \times$ functional group $\times$ performance $(1,3)=0.637$, NS]. Beta power in the piriform cortex followed the same pattern as that seen in the olfactory bulb, with the first session of fine odor discrimination showing enhanced, although not significant, beta power compared with criterion performance $\left[f_{\text {demand }} \times\right.$ performance $(1,3)=7.335, \mathrm{NS}, p=0.073$; $f_{\text {demand }} \times$ functional group $\times$ performance $(1,3)=0.806, \mathrm{NS}]$. Although beta power was
A

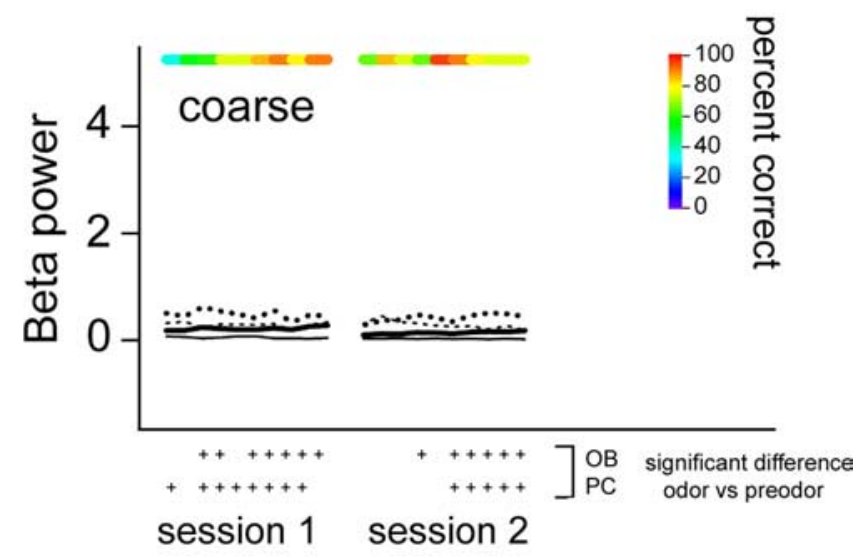

B

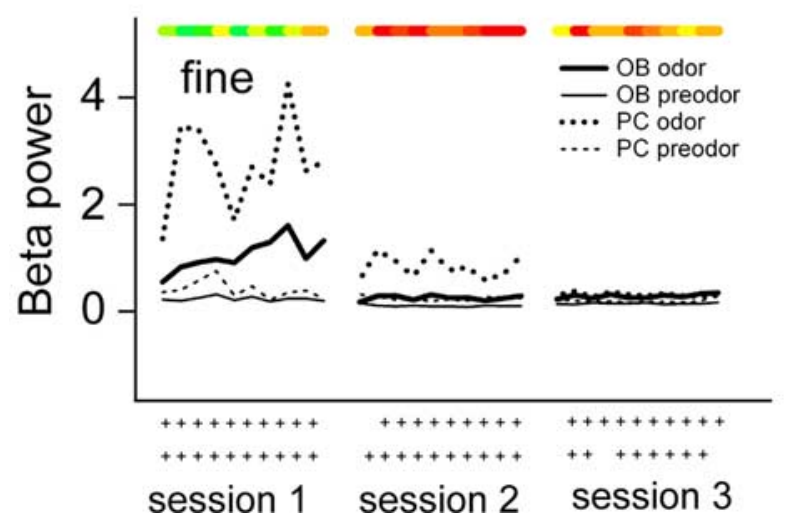

Figure 6. Beta oscillatory power (15-28 Hz) during odor sampling is unrelated to the onset of criterion performance in either fine or coarse odor discrimination (same rat as in Fig. 5). Blockwise performance levels and $p$ values are indicated as in Figure 5. A, Coarse discrimination (propanol/octanol) shows significant elevation in beta power in the olfactory bulb and piriform cortex relative to pre-odor periods but no significant variation across sessions. $\boldsymbol{B}$, Fine odor discrimination (hexanol/heptanol) shows a significant elevation in beta power in the first session, which decreases in the olfactory bulb by the end of the session and remains low thereafter. Piriform cortex beta power stays slightly elevated during the second session, but this pattern is not repeated for ketones or in other rats. Although beta power in the first session of fine discrimination is high for this set of recordings, overall there is only a trend toward an increase in the first session.

slightly greater in both structures during odor sniffing relative to the pre-odor period, there was no selective increase in beta power accompanying criterion performance in either the olfactory bulb or piriform cortex, as there was for gamma (Figs. 5, 6).

\section{Discussion}

Previous studies left open the question of whether fast oscillatory activity represents a functional strategy of olfactory systems across phyla in intact systems during real behavioral circumstances (Stopfer et al., 1997; Nusser et al., 2001). Both of those studies relied on severe disruptions of olfactory system circuitry, and neither study recorded neural activity in animals while they performed odor discriminations. Additionally, as in much of the olfactory psychophysics literature, these studies relied on appetitive reinforcement to a single odorant and tested behavioral generalization to related and unrelated odorants. Recent studies point out that odor discrimination performance and behavioral strategies may in fact be affected by the type of discrimination 
behavior that is required and even the level of reinforcement (Kay et al., 2006; Rinberg et al., 2006). We thus set out to test the oscillation hypothesis in intact, unmanipulated rats performing explicit two-alternative choice odor discriminations. We challenged the rats with multiple odor sets and varied the coarse and fine discrimination variables within each odor class to allow for direct comparisons between the two modes of discrimination.

We show that, in awake, behaving rats performing a twoalternative choice odor discrimination task, olfactory bulb gamma oscillation power is manipulated on-line in a manner that is task demand dependent. This oscillatory activity has been shown to represent precision in firing among olfactory bulb mitral cells, such that higher power indicates a narrower phase of firing by these neurons relative to the underlying gamma oscillation (Gray and Skinner, 1988; Eeckman and Freeman, 1990). The increase in gamma power that accompanies fine odor discrimination, absent from coarse odor discrimination, suggests a functional role of fast oscillatory population activity in the resolution of overlapping odorant stimuli during normal functioning. These enhanced oscillations begin near the end of the sensory evoked potential (Fig. $2 \mathrm{~B}$ ) and persist for $500 \mathrm{~ms}$ or more; this is more time than is required for some odor discriminations (Uchida and Mainen, 2003). However, a recent study has shown that, although fewer sniffs and short sampling times can result in good discrimination performance of relatively nonoverlapping odorant mixtures in a two-alternative choice task, much longer times (>500 ms) are required for highly overlapping odor discriminations (Rinberg et al., 2006). Thus, the duration of these oscillations is within a timeframe that can be directly related to the discrimination process. In the present task, once rats reach criterion levels, performance with odor sets with differing demands is commensurate. However, criterion performance with fine odor sets does correspond to an increase in gamma power. This suggests that upregulation of gamma oscillations may compensate for the discrimination difficulty.

The data also suggest that elevated gamma oscillations are not always necessary (Fig. 5B, downward arrow), because rats can perform well for brief periods in the absence of significantly enhanced gamma for the first block of trials $(\sim 10-20 \mathrm{~min})$ at the beginning of a session in which criterion performance is then sustained for $>1 \mathrm{~h}$. One explanation for the increase in gamma power over the course of criterion performance is that the relatively low levels of gamma power present during the initial trials of criterion performance may simply reflect that very large cooperative assemblies necessary to produce visible changes in the larger-scale LFP are not yet sufficiently organized. However, we must also consider the possibility that, in the mammalian olfactory system, fast oscillatory activity is not strictly or always necessary for fine sensory discrimination. These possibilities point out that the relationship between gamma oscillations and fine odor discrimination may not be as simple for mammals as it appears to be for insects. Alternatively, this may also have been true in the insect studies, because comparison is only possible at the group level; the behavioral methods used in those studies relied on group statistics from single trial performance by large or small numbers of subjects (Stopfer et al., 1997; Nusser et al., 2001). The behavioral data reported by Stopfer et al. (1997) demonstrate that not every animal that was treated with picrotoxin failed to discriminate the fine odorants, but as a group they failed. Similarly, in the $\beta 3$ knock-out mouse, not every epoch of fast sniffing produced significantly enhanced gamma power, nor did every mouse on every trial do better at fine discrimination in separate tests (Nusser et al., 2001). In the present study, when grouped across animals and criterion trials, the data present a clear increase in gamma oscillatory power for fine over coarse discrimination. However, because behavioral and electrophysiological measures were concurrently recorded in our study, we were able to reveal that there is a distinct, albeit not well understood, time course to the increase that is related to criterion performance. There may be multiple ways to solve the problem of fine discrimination in the short term, but some may be more effective for sustained performance. These are issues that deserve additional study to be resolved.

Unlike the relatively short-term evolution of gamma-like odor-evoked oscillations in locusts in response to odor familiarization (Stopfer and Laurent, 1999) or the increases in beta oscillations in rats correlated with learning in a different type of odor discrimination task (Martin et al., 2004), the long-term and selective intensification of gamma power reported here may indicate a specific behavioral or cognitive strategy coupled with a progressive change in network dynamics. The effect does not appear to be a consequence of sensitization. If the results were driven by sensitization through repeated odor sampling, we would expect differences to manifest during the first (naive) session of odor exposure. Gamma power during these sessions is neither elevated (Fig. $4 A, B$ ) nor displays an evolution across the course of the session (Fig. $5 B$, session 1 ). It is only with criterion performance that we see a reliable upregulation of gamma power.

Modulatory and synaptic mechanisms exist in the olfactory bulb that can change the rhythmicity and excitability of the system (Gray and Skinner, 1988; Castillo et al., 1999; Davila et al., 2003) and even possibly discriminability of very similar odorants (Linster and Cleland, 2002). Modulatory effects match the time course of rise in gamma power within a session, taking on the order of 15 min to show sustained effects on behavior (Yue et al., 2004; Pych et al., 2005). The increase in gamma power shown here is similar to that recently reported in the cat visual cortex (Rodriguez et al., 2004). That study showed enhanced gamma oscillations and neural response synchronization that was both delayed and long lasting (on the order of the results reported here), associated with paired cholinergic activation and sensory stimulation in anesthetized animals.

Cholinergic processes are also suggested by the opposite effects on gamma oscillatory power in the olfactory bulb and cortex. We failed to find a related increase in gamma power in the piriform cortex during extremely elevated oscillatory events in the olfactory bulb (Fig. 5), suggesting that these oscillations may be specifically blocked in the piriform cortex during the twoalternative choice task. Although one study of the frog olfactory bulb showed that application of muscarinic cholinergic agonists enhanced odor-evoked oscillations (Hall and Delaney, 2001), theoretical studies of piriform cortex suggest that cholinergic activation there suppresses gamma oscillations (Freeman, 1964, 1968; Liljenstrom and Hasselmo, 1995). Another study has suggested enhanced coupling and high-frequency coherence between the olfactory bulb and piriform cortex after injection of the cholinergic antagonist scopolamine, suggesting that decreasing cholinergic drive couples these two structures more strongly (Chabaud et al., 1999). Future studies will address this hypothesis directly.

The gamma oscillations seen here, during our two-alternative choice task, are distinctly different from the beta oscillations seen in a Go/No-Go task (Martin et al., 2004, 2006). Recent behavioral work has suggested that $\mathrm{Go} / \mathrm{No}-\mathrm{Go}$ and two-alternative choice tasks may use different behavioral strategies for odor discrimination (Uchida and Mainen, 2003; Abraham et al., 2004; Kay et al., 2006; Rinberg et al., 2006), and the difference in oscillatory activ- 
ity shown here suggests that the strategies may involve distinctly different network properties within the olfactory system. Furthermore, the marginally enhanced beta oscillations in the first session of fine discrimination sets may point to a default dynamical strategy that has to be overcome to sustain fine odor discrimination performance in the two-alternative choice task.

The question remains, however, what the strategy that produces large olfactory bulb gamma oscillations provides. Computational models suggest that, for highly overlapping patterns, segmentation is best accomplished with background gamma rhythmicity (Borgers et al., 2005). In olfactory models, fast rhythmic coupling of principal neurons or output neurons have been shown to enhance pattern segmentation (Bazhenov et al., 2001; Linster and Cleland, 2001; Perez-Orive et al., 2004; Sivan and Kopell, 2004; Cleland and Linster, 2005). It is possible that, for sustained performance in this difficult sensorimotor task, it is efficient to use a strong computational strategy to process odors within the $\mathrm{OB}$ circuitry, leaving contextual information for downstream areas.

\section{References}

Abraham NM, Spors H, Carleton A, Margrie TW, Kuner T, Schaefer AT (2004) Maintaining accuracy at the expense of speed: stimulus similarity defines odor discrimination time in mice. Neuron 44:865-876.

Bazhenov M, Stopfer M, Rabinovich M, Abarbanel HDI, Sejnowski TJ, Laurent G (2001) Model of cellular and network mechanisms for odorevoked temporal patterning in the locust antennal lobe. Neuron 30:569-581.

Borgers C, Epstein S, Kopell NJ (2005) Background gamma rhythmicity and attention in cortical local circuits: a computational study. Proc Natl Acad Sci USA 102:7002-7007.

Bower JM, Haberly LB (1986) Facilitating and nonfacilitating synapses on pyramidal cells: a correlation between physiology and morphology. Proc Natl Acad Sci USA 83:1115-1119.

Bressler SL, Freeman WJ (1980) Frequency analysis of olfactory system EEG in cat, rabbit, and rat. Electroencephalogr Clin Neurophysiol 50:19-24.

Castillo PE, Carleton A, Vincent JD, Lledo PM (1999) Multiple and opposing roles of cholinergic transmission in the main olfactory bulb. J Neurosci 19:9180-9191.

Chabaud P, Ravel N, Wilson DA, Gervais R (1999) Functional coupling in rat central olfactory pathways: a coherence analysis. Neurosci Lett 276:17-20.

Cleland TA, Linster C (2002) How synchronization properties among second-order sensory neurons can mediate stimulus salience. Behav Neurosci 116:212-221.

Cleland TA, Linster C (2005) Computation in the olfactory system. Chem Senses 30:801-813.

Davila NG, Blakemore LJ, Trombley PQ (2003) Dopamine modulates synaptic transmission between rat olfactory bulb neurons in culture. J Neurophysiol 90:395-404.

Eeckman FH, Freeman WJ (1990) Correlations between unit firing and EEG in the rat olfactory system. Brain Res 528:238-244.

Freeman WJ (1964) Linear distributed feedback model for prepyriform cortex. Exp Neurol 10:525-547.

Freeman WJ (1968) Effects of surgical isolation and tetanization on prepyriform cortex in cats. J Neurophysiol 31:349-357.

Friedrich RW, Laurent G (2001) Dynamic optimization of odor representations by slow temporal patterning of mitral cell activity. Science 291:889-894.

Friedrich RW, Habermann CJ, Laurent G (2004) Multiplexing using synchrony in the zebrafish olfactory bulb. Nat Neurosci 7:862-871.

Gray CM, Skinner JE (1988) Centrifugal regulation of neuronal activity in the olfactory bulb of the waking rabbit as revealed by reversible cryogenic blockade. Exp Brain Res 69:378-386.

Hall B, Delaney K (2001) Cholinergic modulation of odor-evoked oscillations in the frog olfactory bulb. Biol Bull 201:276-277.

Homanics GE, DeLorey TM, Firestone LL, Quinlan JJ, Handforth A, Harrison NL, Krasowski MD, Rick CE, Korpi ER, Makela R, Brilliant MH, Hagiwara N, Ferguson C, Snyder K, Olsen RW (1997) Mice devoid of gamma-aminobutyrate type A receptor beta3 subunit have epilepsy, cleft palate, and hypersensitive behavior. Proc Natl Acad Sci USA 94:4143-4148.

Johnson BA, Farahbod H, Xu Z, Saber S, Leon M (2004) Local and global chemotopic organization: general features of the glomerular representations of aliphatic odorants differing in carbon number. J Comp Neurol 480:234-249.

Kay LM (2003) Two species of gamma oscillations in the olfactory bulb: dependence on behavioral state and synaptic interactions. J Integr Neurosci 2:31-44.

Kay LM (2005) Theta oscillations and sensorimotor performance. Proc Natl Acad Sci USA 102:3863-3868.

Kay LM, Freeman WJ (1998) Bidirectional processing in the olfactorylimbic axis during olfactory behavior. Behav Neurosci 112:541-553.

Kay LM, Beshel J, Martin C (2006) When good enough is best. Neuron 51:277-278.

Klingberg F, Pickenhain L (1965) Uber langsame atemsynchrone Potentiale vom Bulbus olfactorius der Ratte. Acta Biol Med Ger 14:593-595.

Konig P, Engel AK, Singer W (1995) Relation between oscillatory activity and long-range synchronization in cat visual-cortex. Proc Natl Acad Sci USA 92:290-294.

Liljenstrom H, Hasselmo ME (1995) Cholinergic modulation of cortical oscillatory dynamics. J Neurophysiol 74:288-297.

Linster C, Cleland TA (2001) How spike synchronization among olfactory neurons can contribute to sensory discrimination. J Comput Neurosci 10:187-193.

Linster C, Cleland TA (2002) Cholinergic modulation of sensory representations in the olfactory bulb. Neural Networks 15:709-717.

Linster C, Johnson BA, Yue E, Morse A, Xu Z, Hingco EE, Choi Y, Choi M, Messiha A, Leon M (2001) Perceptual correlates of neural representations evoked by odorant enantiomers. J Neurosci 21:9837-9843.

Macrides F, Chorover SL (1972) Olfactory bulb units: activity correlated with inhalation cycles and odor quality. Science 175:84-87.

Martin C, Gervais R, Hugues E, Messaoudi B, Ravel N (2004) Learning modulation of odor-induced oscillatory responses in the rat olfactory bulb: a correlate of odor recognition? J Neurosci 24:389-397.

Martin C, Gervais R, Messaoudi B, Ravel N (2006) Learning-induced oscillatory activities correlated to odour recognition: a network activity. Eur J Neurosci 23:1801-1810.

Mori K, Mataga N, Imamura K (1992) Differential specificities of single mitral cells in rabbit olfactory bulb for a homologous series of fatty acid odor molecules. J Neurophysiol 67:786-789.

Nusser Z, Kay LM, Laurent G, Homanics GE, Mody I (2001) Disruption of $\mathrm{GABA}(\mathrm{A})$ receptors on GABAergic interneurons leads to increased oscillatory power in the olfactory bulb network. J Neurophysiol 86:2823-2833.

Perez-Orive J, Mazor O, Turner GC, Cassenaer S, Wilson RI, Laurent G (2002) Oscillations and sparsening of odor representations in the mushroom body. Science 297:359-365.

Perez-Orive J, Bazhenov M, Laurent G (2004) Intrinsic and circuit properties favor coincidence detection for decoding oscillatory input. J Neurosci 24:6037-6047.

Pych JC, Chang Q, Colon-Rivera C, Gold PE (2005) Acetylcholine release in hippocampus and striatum during testing on a rewarded spontaneous alternation task. Neurobiol Learn Mem 84:93-101.

Rinberg D, Koulakov A, Gelperin A (2006) Speed-accuracy tradeoff in olfaction. Neuron 51:351-358.

Rodriguez R, Kallenbach U, Singer W, Munk MHJ (2004) Short- and longterm effects of cholinergic modulation on gamma oscillations and response synchronization in the visual cortex. J Neurosci 24:10369-10378.

Sivan E, Kopell N (2004) Mechanism and circuitry for clustering and fine discrimination of odors in insects. Proc Natl Acad Sci USA 101:17861-17866.

Stopfer M, Laurent G (1999) Short-term memory in olfactory network dynamics. Nature 402:664-668.

Stopfer M, Bhagavan S, Smith BH, Laurent G (1997) Impaired odour discrimination on desynchronization of odour-encoding neural assemblies. Nature 390:70-74.

Uchida N, Mainen ZF (2003) Speed and accuracy of olfactory discrimination in the rat. Nat Neurosci 6:1224-1229.

Yue EL, Cleland TA, Pavlis M, Linster C (2004) Opposing effects of D-1 and D-2 receptor activation on odor discrimination learning. Behav Neurosci 118:184-190. 